\title{
Connectivity Analysis of Underwater Optical Wireless Sensor Networks
}

\author{
Reza Habibiyan, and Abbasali Ghorban Sabbagh, Member, IEEE
}

\begin{abstract}
This paper analyses the connectivity of the underwater optical wireless sensor networks in which similar nodes with a certain range and coverage angle, and random surface distribution are used. In the analyses of this paper, nodes are first classified into two categories: nodes located in the inner area and nodes located in the border strip, and the analysis of their connectivity is presented separately. By combining the mentioned analyses, we achieve closed form formulas to calculate the probability of connectivity (from order 1 and 2) in these networks, these formulas provide the possibility of predicting the average probability of network connectivity as a function of number, communication range, and coverage angle of optical node. Furthermore, by numerical evaluation, we show how much the results of the analyses become closer to the results of computer simulation by considering the effect of nodes located in border strip compared to previous works.
\end{abstract}

Index Terms-Underwater wireless optical sensor networks, probability of node connectivity, border strip.

\section{INTRODUCTION}

$\mathrm{R}$ ECENTLY, there has been a strong interest in studying the underwater environment for various applications, such as studying climate changes, marine biology, monitoring of oil rigs, and unmanned operations. These programs require a media for connection from the underwater environment to the environment outside the water [1].

Underwater acoustic wireless communication (UAWC) provides communication over very long distances (several kilometers) [2]; however, the nominal propagation speed of underwater acoustic waves is around $1500 \mathrm{~m} / \mathrm{s}$ and, the operational bandwidth of the underwater acoustic signal is between tens of $\mathrm{Hz}$ to hundreds of $\mathrm{kHz}$. The achievable data rate of acoustic links is at most in the range of kilo bit per second, which is not suitable for high-rate data transmission [3]. In addition, the use of acoustic waves has disadvantages such as scattering, long delays due to low propagation speed, and adverse effects on fishes and marine mammals [4]. To solve this problem, low radio frequency (RF) systems were introduced, however they had limited data rate and short communication range [5]. In addition, low-frequency RF-based underwater wireless communication requires large antennas and is limited to shallow sea areas. On the other hand, operation at low frequencies reduces efficiency and causes high hardware costs and low data rate [6]. Underwater RF systems have a much higher propagation speed than underwater acoustic systems but suffer from very high attenuation, especially for the frequency range of about $\mathrm{MHz}$ and higher [7].

An alternative method to the RF system due to its limitations could be optical waves because underwater optical wireless communications (UOWC) can transmit data at higher rates with a propagation speed of about $2.25 \times 10^{8}$ $\mathrm{m} / \mathrm{s}$. However, phenomena such as absorption and scat-

- R. Habibiyan and A. Ghorban Sabbagh are with the Department of Electrical and Computer Engineering, Quchan University of Technology, Quchan, 94771-67335, Iran.

E-mail:a.ghorbansabbagh@qiet.ac.ir (Corresponding author) tering raise significant challenges to increase the communication range of the underwater optical wireless network (UOWN) [8], [9]. In addition to absorption and scattering, another destructive phenomenon that affects the underwater optical communication range is optical turbulence. Optical turbulence is caused by random changes of refractive index along the transmitted beam path; Factors affecting the random changes include variation in temperature, pressure, salinity, etc. [10], [11]. Accordingly, some researches have been done to increase the transmission range of UOWC using relays [12], [13]. Furthermore, to improve the robustness of the UOWC, using the multi-input multi-output (MIMO) technique has also been proposed [14]. Along with the efforts made to overcome the obstacles on employing the optical communication in underwater environments, the idea of combining acoustic and optical methods is also proposed; see reference [15] for example.

It should be noted that the realization of light transmitters and receivers using lasers and photo diodes (PD) in small dimensions, with low cost and low energy consumption is a significant advantage of the optical method compared to other methods. In addition, a new type of highly sensitive optical receiver called single photon avalanche diode (SPAD) has recently been introduced in applications of underwater optical communications, and the communication range of up to about 500 meters for pure seawater has also been reported with the help of computer simulation [16]. It has been shown that the least attenuation of light waves in the water environment is in the range of $450-550$ nm [17].

Practically, one of the most important concepts about a wireless sensor network (WSN) is the concept of network connectivity. Important parameters of an optical sensor network such as number of nodes, node arrangement, communication range (power consumption) of nodes, coverage angle of nodes and, their orientation are the factors affecting the connectivity of a such network. The existence of these parameters makes the connection of the network have a 
probabilistic concept. The probabilistic nature of connectivity for optical wireless networks becomes more evident when in many applications, the location of the nodes is random, and in addition, the nodes are also directed (the connection of the node with other nodes depends on the orientation of the node). It should be noted that optical nodes, unlike RF-equipped nodes, are not omnidirectional and usually emit light around a certain direction [18]. Accordingly, the connectivity analysis in UOWN consists of more serious challenges than RF sensor networks. Generally, network connectivity analysis (calculating the probability that a node is not blocked) has different applications, and one of its most important is the network nodes localization [19]. Localization in terrestrial networks has been extensively studied, and the proposed techniques have reached acceptable maturity. However, global positioning system (GPS) and RF-based designs cannot be used in the underwater environment [19]. Localization in underwater acoustic networks has been thoroughly studied in [19], but generally, it is not possible to use localization techniques in wireless ground networks and underwater acoustic networks for UOWNs. For UOWNs, distributed localization schemes based on the time of arrival (ToA) and received signal strength (RSS), and centralized localization schemes based on RSS have been presented [20], [21], [22]. In [23] an RSSbased centralized localization system has been introduced, in which the estimated single-hop distances of neighbors from each underwater optical sensor node are collected by the surface station, and then it estimates the location of the nodes using at least three anchor nodes. Then, using the matrix completion strategy, it estimates missing pairwise distances.

In this paper, we analyze the connectivity in the UOWN in which similar nodes with a certain range and coverage angle, and random surface distribution are used. In our analysis, we first classify the nodes into two categories of nodes located in the inner area and nodes located in the border strip by defining the width of the border strip as the node range. Then, we conduct their connectivity analyses separately. Finally, by combining the mentioned analyses, we obtain closed form formulas to calculate the probability of connectivity (from orders 1 and 2). Moreover, by numerical evaluation, we will compare the results of the analyses with the results of computer simulation.

The remainder of this article is as follows. Section 2 provides a brief review of underwater optical wireless netwroks model. Our connectivity analyses are thoroughly conducted in Section 3. Section 4 is devoted to present the numerical results and simulation, and finally, Section 5 concludes the paper.

\section{Underwater Optical Wireless Networks MODEL}

\subsection{Channel Model}

Absorption is the most crucial factor in limiting the range of underwater optical wireless networks and causes the beam energy to be continuously reduced along the propagation path. Scattering, on the other hand, deflects photons in random directions so that some of them are not received due to the limited size of the receiver aperture; while receiving some other parts may be delayed due to the longer path to reach the receiver aperture. Scattering therefore, leads to multipath fading, temporal jitter, and inter symbol interference (ISI). The sum of coefficients of absorption $a(\lambda)$ and scattering $b(\lambda)$ is called the extinction coefficient $c(\lambda)$ and, we have:

$$
c(\lambda)=a(\lambda)+b(\lambda)
$$

which all are a function of wavelength $(\lambda)$.

\subsubsection{Attenuation}

Beer Lambert law: it is the simplest model for describing attenuation (expressing the relationship between received and transmitted signal power) at UOWC and is widely used. According to this law, we have [16]:

$$
P_{r}=P_{t} e^{-c(\lambda) d}
$$

where $P_{r}$ is the received power, $P_{t}$ is the transmitted power and $d$ is the distance between the transmitter and the receiver. Beam Lambert's law assumes that scattered photons have no chance of being received by the receiver. In practice, the range of an optical connection depends on parameter $c$ and varies for different types of water [24].

\subsubsection{Turbulence}

Underwater optical channel modeling focuses mostly on the effects of absorption and scattering, and there is not much study on the effect of refractive index changes called optical turbulence on system performance. The mechanisms of atmospheric light turbulence and ocean light turbulence have significant similarities, so some studies of oceanic light turbulence modeling have been adapted from free space optical (FSO) models. Weak turbulence is well modeled by Log-normal distribution as follows [25]:

$$
f_{I}(I)=\frac{1}{I \sigma \sqrt{2 \pi}} e^{-\left(\ln \left(\frac{I}{I_{0}}\right)-\mu\right)^{2} /\left(2 \sigma^{2}\right)}
$$

where $I$ is the received light intensity and $I_{0}$ is its mean, $\mu$ is the mean of the logarithm of the light intensity, and $\sigma$ is the scintillation index. In the case of weak oceanic turbulence, an aperture averaging method has been investigated and it has been shown that system performance increases by decreasing the scintillation index. In addition, turbulence has been shown to have a major effect on temporal correlation of irradiance, while the link length has less effect [26].

\subsection{Network Model}

Wireless optical networks are directed, unlike omnidirectional acoustic and radio networks. Suppose we have $M$ same nodes in the optical network and we have numbered them with the index $i=1,2,3, \ldots, M$. In addition, $\nu_{i}$ is the random orientation of the node relative to the horizontal axis, $\varphi_{i}$ is coverage angle of the node, $R_{i}$ is the node range, and $c_{i}$ is the node coordinate (see Fig. 1a). The random sector directed graph can be shown as $\mathcal{G}(\mathcal{V}, \mathcal{E})$ where $\mathcal{V}=\left(c_{1}, c_{2}, \ldots, c_{M}\right)$ is coordinates of nodes (graph vertices) and $\mathcal{E}=\{0,1\}^{M}$ is lines of graph. The presence of node $j$ in the area covered by node $i$ is denoted by $n_{i} \rightarrow n_{j}$. This can also be expressed as $\mathcal{E}_{i, j}=1$. Set of descendant nodes of node $i\left(n_{i}\right)$ are shown with $\mathcal{D}_{i}=\left\{n_{j} \mid \forall j: \mathcal{E}_{i, j}=1\right\}$ 


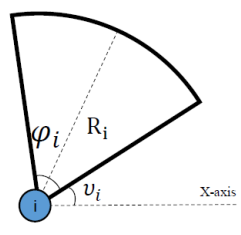

(a)

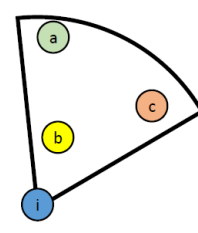

(b)

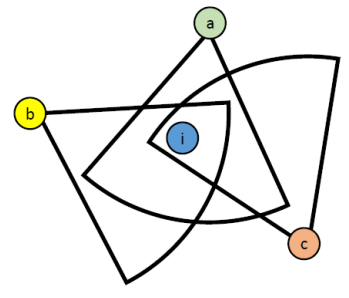

(c)
Fig. 1: (a) Parameters of node $i$, (b) descendents of node $i$, (c) antecendents of node $i$.

(see Fig. 1b) and the set of antecedent of nodes $\left(n_{i}\right)$ with $\mathcal{A}_{i}=\left\{n_{j} \mid \forall j: \mathcal{E}_{j . i}=1\right\}$ (see Fig. 1c). We show the cardinality of the sets $\mathcal{D}_{i}$ and $\mathcal{A}_{i}$ with $D_{i}$ and $A_{i}$, respectively. The random variables $D_{i}$ and $A_{i}$ play an important role in network connectivity analysis as we will see in the next section. By definition, a network in which each node has at least one descendant node and one antecedent node is called a 1-connected network.

\section{Connectivity Analysis in Underwater Op- TICAL WIRELESS NETWORKS}

If $D_{i}\left(A_{i}\right)$ is the number of nodes of the descendant (antecedent), then the probability of having a node of the descendant (antecedent) can be expressed as $p_{d}^{i}=\operatorname{Pr}\left[D_{i} \geq 1\right]$ $\left(p_{a}^{i}=\operatorname{Pr}\left[A_{i} \geq 1\right]\right)$. Accordingly, the probability of node connectivity for the $i$-th node can be expressed as $p_{c n}^{i}=$ $p_{d}^{i} \times p_{a \mid d}^{i}$, that in this relation $p_{a \mid d}^{i}$ is the probability that $n_{i}$ has antecedent nodes, given that this node has descendant node(s). In [23] and [27], the effect of nodes located in the border area (assumed to be a square of the side $\alpha$ ) has been mentioned, but its effect has not been considered in their analyses. In this paper, considering the effect of nodes located at the boundaries, the average probability of the nodes connectivity is calculated. For this purpose, we divide the distribution area of the node based on the node range and, according to Fig. 2, divide it into two inner area and the border strip, and calculate the probability of connectivity of a node located in each of these areas. As can be seen, the border strip has a width of $R$ that is equal to the range of all nodes. The reason for choosing the value of $R$ to define the border strip is that if the node is not in this strip, regardless of the orientation of the node, its sector (coverage area) is located inside the area of node distribution, which is assumed to be a square of the side $\alpha$. However, for nodes located in the border area, depending on the orientation of the node, some parts of the sector may not be in the distribution area of the nodes. In addition, Fig. 2 shows an area in the border strip that is spaced (horizontally or vertically) $\beta$ relative to the inner area's border.

For node $n_{i}$, the probability of connectivity can be expressed as follows:

$$
p_{c n}^{i}=\left(p_{c n}^{i}\right)_{i n} \operatorname{Pr}\left(n_{i} \in A_{i n}\right)+\left(p_{c n}^{i}\right)_{b o} \operatorname{Pr}\left(n_{i} \in A_{b o}\right)
$$

where $\left(p_{c n}^{i}\right)_{i n}$ and $\left(p_{c n}^{i}\right)_{b o}$ indicate the probability of connectivity of a node located in the inner and border area,

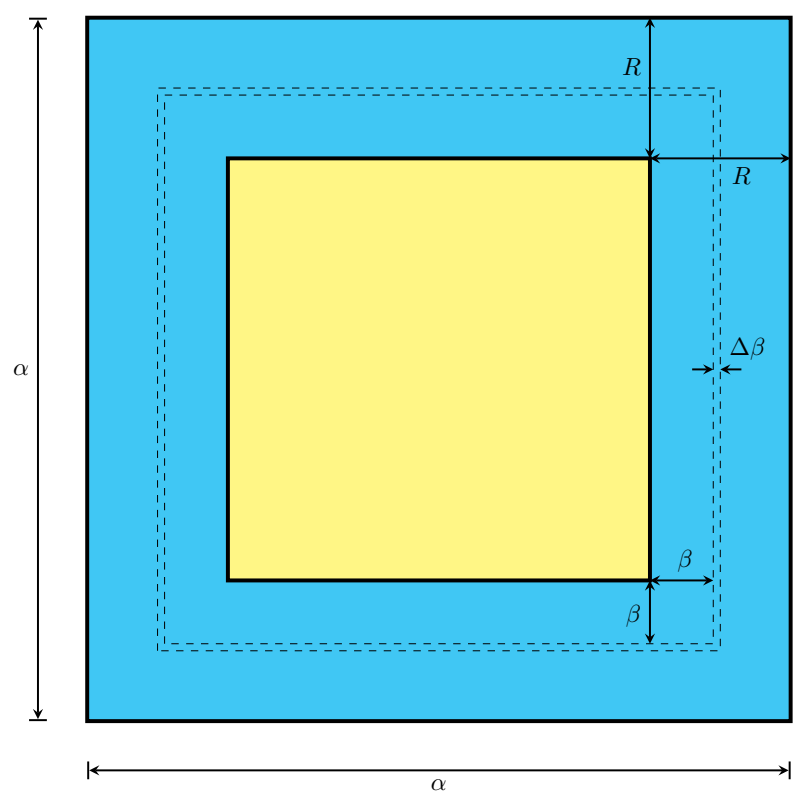

Fig. 2: Partitioning the area of nodes distribution into two areas; inner (yellow color) and border strip (blue color).

respectively, and $A_{i n}=(\alpha-2 R)^{2}$ and $A_{b o}=4 R(\alpha-R)$ represent the inner and border surface areas, respectively.

In order to simplify and make the analysis independent of the dimensions of the area, we will use the normalized parameters. Accordingly, we represent the normalized range of each node as $r=R / \alpha$. According to the geometry considered for the problem, the value of $R$ changes in the range $0<R \leq \frac{\alpha}{2}$ and, as a result we have $0<r \leq \frac{1}{2}$. By using the normalized parameters, the probability of a node locating in inner area and the border strip are: $(1-2 r)^{2}$ and $4 r-4 r^{2}$, respectively. Next, we show the normalized $\beta$ with $\epsilon=\beta / \alpha$. Fig. 3 shows the normalized area of nodes distribution as well as its partitioning into four blue side rectangles and four white corner squares.

\subsection{Connectivity Analysis in the Inner Area}

In this subsection, we calculate the probability of a node being connected that is located in the inner area. For this purpose, we assume that $i$ is a node located in the inner area. Probability of connectivity for node $i$ is as follows:

$$
\left(p_{c n}^{i}\right)_{i n}=\left(p_{d}^{i} \times p_{a \mid d}^{i}\right)_{i n} .
$$

Considering the normalized node distribution area as a unit square, and assuming a large number of nodes, and a small normalized range (i.e. $M \rightarrow \infty$ and $r \ll 1$ ), the distribution of nodes can be well modeled by homogeneous Poisson point process with $Q=\frac{M r^{2} \varphi}{2}$ and we have:

$$
p_{d}^{i}=p_{a}^{i}=1-e^{-Q} .
$$

Next, to calculate $p_{a \mid d}^{i}$, we do the following:

$$
\begin{aligned}
p_{a \mid d}^{i} & =1-\operatorname{Pr}\left(A_{i}=0 \mid D_{i} \geq 1\right) \\
& =1-\frac{\operatorname{Pr}\left(A_{i}=0, D_{i} \geq 1, \gamma=0\right)}{\operatorname{Pr}\left(D_{i} \geq 1\right)} .
\end{aligned}
$$




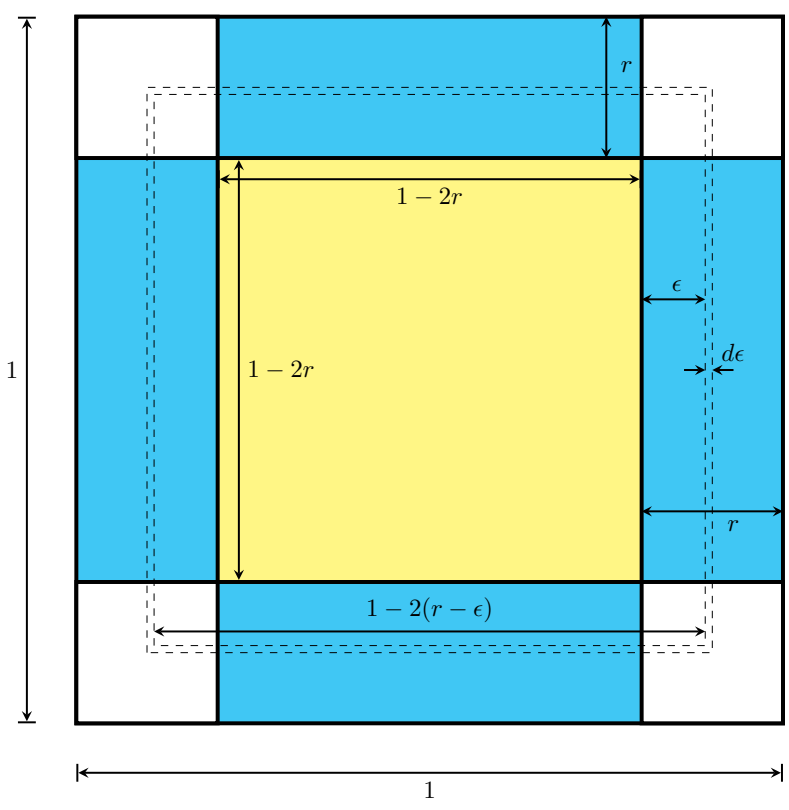

Fig. 3: Partitioning the normalized border strip into four blue side rectangles and four white corner squares.

In this relation, $\gamma$ represents the number of bidirectional links that node $i$ has with its descendant. Equation (7) can be expressed as follows:

$$
\begin{array}{r}
p_{a \mid d}^{i}=1-\frac{1}{\operatorname{Pr}\left(D_{i} \geq 1\right)} \sum_{b=1}^{M-1} \operatorname{Pr}\left(A_{i}=0 \mid D_{i}=b, \gamma=0\right) \times \\
\operatorname{Pr}\left(\gamma=0 \mid D_{i}=b\right) \operatorname{Pr}\left(D_{i}=b\right) .
\end{array}
$$

According to the geometry of the problem, the probabilities in relation (8) are calculated as described below. Assuming the Poisson distribution nodes, the probability that node $i$ (located in the inner area) has $b$ descendant nodes is equal to:

$$
\operatorname{Pr}\left(D_{i}=b\right)=\frac{e^{-Q} Q^{b}}{b !} .
$$

Assuming that node $i$ has $b$ descendants, the probability of not having a bidirectional link between node $i$ and its descendants are calculated as below:

$$
\operatorname{Pr}\left(\gamma=0 \mid D_{i}=b\right)=\left(1-\frac{\varphi}{2 \pi}\right)^{b} .
$$

To calculate the expression $\operatorname{Pr}\left(A_{i}=0 \mid D_{i}=b, \gamma=0\right)$ in (8), we define the random variable ${ }_{i} D$ as the number of nodes located in the complementary area of the node $i$ sector, i.e. the complementary sector of the node $i$ sector. As the total number of nodes is $M$ and the number of descendants of node $i$ is assumed to be $b$, the random variable ${ }_{i} D$ can select its values from the set $\{0,1, \ldots, M-1-b\}$. Of the total $M$ nodes, some are inside the circle with the center of node $i\left(C_{i}\right)$ and the rest are outside it. It is evident that nodes outside $C_{i}$ cannot be the antecedent of node $i$, and only ${ }_{i} D$ nodes in the complementary area of the sector of $i$ will have a chance of being the antecedent of node $i$. It should be noted that in this paper, the calculation of the expression $\operatorname{Pr}\left(A_{i}=0 \mid D_{i}=b, \gamma=0\right)$ is done more accurately than the references [23] and [27].
The distribution of nodes in the complementary sector can be considered by the Poisson distribution with an average of $Q^{\prime}=\frac{M r^{2}(2 \pi-\varphi)}{2}$.

$$
\begin{aligned}
\operatorname{Pr}\left(A_{i}=0 \mid D_{i}=b, \gamma=0\right) & \\
= & \sum_{N=0}^{M-1-b} \operatorname{Pr}\left(A_{i}=0,{ }_{i} D=N \mid D_{i}=b, \gamma=0\right) \\
= & \sum_{N=0}^{M-1-b} \operatorname{Pr}\left(A_{i}=\left.0\right|_{i} D=N, D_{i}=b, \gamma=0\right) \\
& \quad \times \operatorname{Pr}\left({ }_{i} D=N \mid D_{i}=b, \gamma=0\right) .
\end{aligned}
$$

On the other hand, assuming the event $\left(A_{i}=\left.0\right|_{i} D=N\right)$ and $\left(D_{i}=b, \gamma=0\right)$ are independent, we have:

$$
\operatorname{Pr}\left(A_{i}=\left.0\right|_{i} D=N, D_{i}=b, \gamma=0\right)=\left(1-\frac{\varphi}{2 \pi}\right)^{N} .
$$

In addition, assuming the events $\left({ }_{i} D=N\right)$ and $\left(D_{i}=\right.$ $b, \gamma=0)$ are independent, for $N=0,1, \ldots, M-1-b$ we have:

$$
\operatorname{Pr}\left({ }_{i} D=N \mid D_{i}=b, \gamma=0\right)=\operatorname{Pr}\left({ }_{i} D=N\right)=\frac{e^{-Q^{\prime}} Q^{\prime N}}{N !} .
$$

Finally, by substituting the obtained expressions in (8), we will have:

$$
\begin{aligned}
& p_{a \mid d}^{i}= \\
& 1-\frac{\sum_{b=1}^{M-1} \sum_{N=0}^{M-1-b}\left[\left(1-\frac{\varphi}{2 \pi}\right)^{N} \frac{e^{-Q^{\prime}} Q^{\prime N}}{N !}\right]\left[\left(1-\frac{\varphi}{2 \pi}\right)^{b} \frac{e^{-Q} Q^{b}}{b !}\right]}{1-e^{-Q}} \\
& =1-\frac{e^{-M \pi r^{2}}}{1-e^{-Q}} \sum_{b=1}^{M-1} \sum_{N=0}^{M-1-b} \frac{\left[\left(1-\frac{\varphi}{2 \pi}\right) Q^{\prime}\right]^{N}}{N !} \frac{\left[\left(1-\frac{\varphi}{2 \pi}\right) Q\right]^{b}}{b !} .
\end{aligned}
$$

In the last equation, the relation $Q+Q^{\prime}=M \pi r^{2}$ is used. Therefore, $\left(p_{c n}^{i}\right)_{i n}$ for nodes in the inner area is obtained using (5) as follows:

$$
\begin{aligned}
& \left(p_{c n}^{i}\right)_{i n}=\left(1-e^{-Q}\right) \times \\
& \left(1-\frac{e^{-M \pi r^{2}}}{1-e^{-Q}} \sum_{b=1}^{M-1} \sum_{N=0}^{M-1-b} \frac{\left[\left(1-\frac{\varphi}{2 \pi}\right) Q^{\prime}\right]^{N}}{N !} \frac{\left[\left(1-\frac{\varphi}{2 \pi}\right) Q\right]^{b}}{b !}\right) .
\end{aligned}
$$

\subsection{Connectivity Analysis in the Border Strip}

In this subsection, we calculate the probability of connectivity of a node located in the border strip. For this purpose, we assume that node $i$ is a node located in the border strip; therefore, we indicate the probability of connectivity of this node as $\left(p_{c n}^{i}\right)_{b o}$, and state it as:

$$
\left(p_{c n}^{i}\right)_{b o}=\left(p_{d}^{i} \times p_{a \mid d}^{i}\right)_{b o} .
$$

As mentioned in the previous section, the sector of nodes located in the inner area is entirely (with a unit coefficient $\chi=1$ ) inside the unit square, regardless of its orientation; however, part of the sector of nodes located in the border strip may not be inside the unit square. The question that arises here and we are looking for the answer in this section is that what is the average of the coverage of the sector of the node located in the border strip? We denote this coefficient, 
which is less than one, by $\chi$, and will refer to it as the coefficient which correlates $\bar{Q}$ (average of Poisson distribution of nodes in the border area) to $Q$ and also $\bar{Q}^{\prime}$ (average of Poisson distribution of nodes in the complementary border area) to $Q^{\prime}$, which means $\bar{Q}=\chi Q$ and $\bar{Q}^{\prime}=\chi Q^{\prime}$. In other words, the coefficient $\chi$ indicates that, regarding the presence of a node in the border strip, on average, what proportion of the node sector overlap with the unit square area. According to the geometry of the problem, the border strip is comprised of the four blue side rectangles in Fig. 3. In addition, the border strip includes four white corner squares.

To simplify the calculations, we begin our study to determine the coefficient $\chi$ for a node with normalized range $r$ and angle $\varphi=2 \pi$. The angle $2 \pi$ was chosen here because that the calculations do not depend on the node orientation parameter (parameter $\nu_{i}$ in Fig. 1). In addition, in order to simplify the calculations in this paper, in examining the nodes located in the border strip, we assume that the node is not located in the four side squares. It is evident that by decreasing $r$, the approximation of this assumption decreases, and our analyses become more accurate. Appendix A shows that for $\varphi=2 \pi$ we have:

$$
\chi=1-\frac{1}{\pi}\left(\arccos \left(1-\frac{\epsilon}{r}\right)\right)+\frac{r-\epsilon}{\pi r^{2}} \sqrt{2 \epsilon r-\epsilon^{2}} .
$$

As can be seen, $\chi$ is a function of the variables $\epsilon$ and $r$. In the following, we will calculate the expectation of $\chi$ relative to $\epsilon$, denoted by $\bar{\chi}$. In other words, we want to know, regardless of the position of the node in the border area, on average, what proportion of the area covered by the node is inside the unit square. For this purpose, we continue our calculations as follows:

$$
\begin{aligned}
& \bar{\chi}= E_{\epsilon}[\chi]=\int_{0}^{r} \chi f_{\epsilon}(\epsilon) d \epsilon \\
&=\int_{0}^{r}\left(1-\frac{1}{\pi}\left(\arccos \left(1-\frac{\epsilon}{r}\right)\right)+\frac{r-\epsilon}{\pi r^{2}} \sqrt{2 \epsilon r-\epsilon^{2}}\right) \\
& \quad \times\left(\frac{1-2(r-\epsilon)}{r-r^{2}}\right) d \epsilon \\
&=1+\int_{0}^{r}\left(\frac{r-\epsilon}{\pi r^{2}} \sqrt{2 \epsilon r-\epsilon^{2}}-\frac{1}{\pi}\left(\arccos \left(1-\frac{\epsilon}{r}\right)\right)\right) \\
& \quad \times\left(\frac{1-2(r-\epsilon)}{r-r^{2}}\right) d \epsilon \\
&=1+\frac{-6 \pi r^{2}-8 r+3 r(8+\pi r)}{24 \pi r(r-1)} .
\end{aligned}
$$

In the calculations of (18), the probability density function $f_{\epsilon}(\epsilon)$ calculated in Appendix A is used. The closed form relation obtained in (18) is obtained only for $\varphi=2 \pi$ and where the node is assumed to be located in the four side rectangles of the border strip. Since the calculation of $\bar{\chi}$ without the proposed assumption (i.e., ignoring the four squares in corners) will increase the complexity of the calculations, and on the other hand, it is necessary to measure the accuracy of the calculations, a computer simulation is used to estimate the $\bar{\chi}$ by calculating the four squares of the border strip. For this purpose, to obtain $\bar{\chi}$, we produce 20,000 nodes with a coverage angle of $2 \pi$ and a uniform distribution in the border strip, and then estimate the value of $\bar{\chi}$ by determining the $\chi$ of each node and then averaging

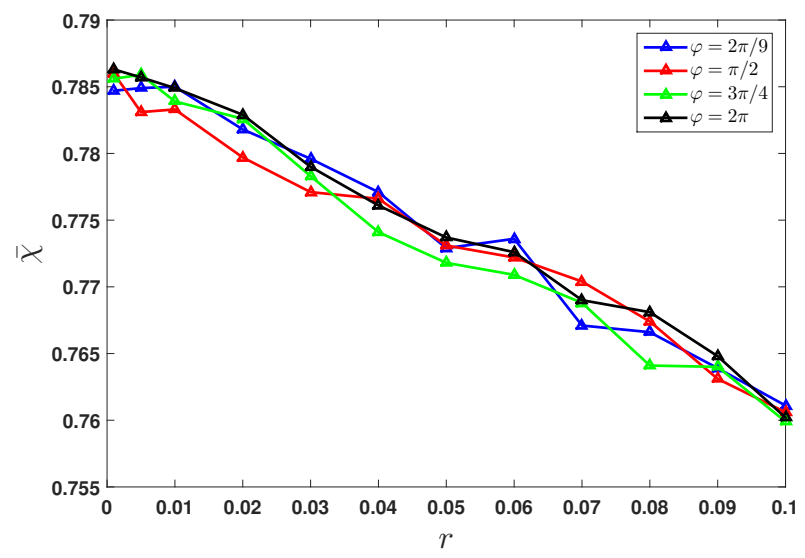

Fig. 4: $\bar{\chi}$ obtained from simulation vs. $r$ for coverage angles of $2 \pi / 9, \pi / 2,3 \pi / 4$, and $2 \pi$.

them. The results of obtained $\bar{\chi}$ from (18) and computer simulation for different values of $r$ and coverage angle $2 \pi$ are given in Table 1.

Comparison of the results obtained from Table 1 shows that, as expected, the results of $\bar{\chi}$ obtained by the analytical method are higher than $\bar{\chi}$ obtained by simulation due to the lack of calculation of the four corners of the unit square in the border areas. It should also be noted that, as predicted, for small $r \mathrm{~s}$, the analysis results and the simulation results are very close. The results of Table 1 show that using (18) to calculate $\bar{\chi}$ has acceptable accuracy and the approximation performed to calculate this relation, especially when $r$ is small, has minimal effect on the results. In addition, the results in Table 1 show as the values of $r$ increases, the value of the coefficient $\bar{\chi}$ decreases, but the amount of this decrease is rather small.

Since obtaining the analytical relation of $\bar{\chi}$ for nodes located in the border strip with an angle other than $2 \pi$ requires complex averaging on the orientation of the node $\left(\nu_{i}\right.$ in Fig. $1)$, and the results obtained from computer simulation for $\varphi=2 \pi$ is very close to the analytical results; in the this paper, the value of $\bar{\chi}$ for the angles $2 \pi / 9, \pi / 2,3 \pi / 4$ is simply determined through simulation, as described earlier.

The simulation results for $\bar{\chi}$ and for the coverage angles $2 \pi / 9, \pi / 2,3 \pi / 4$, and $2 \pi$ are shown in Fig. 4 . As can be seen in this figure, the value obtained for $\bar{\chi}$ is similar to the results obtained for $2 \pi$, in the range of $0.76-0.78$, and has

TABLE 1: Comparison of $\bar{\chi}$ obtained form analytical and simulation methods for different values of $r$ and for $\varphi=2 \pi$

\begin{tabular}{ccc}
\hline Normalized range $r$ & Analytical $\bar{\chi}$ from $(18)$ & $\bar{\chi}$ from simulation \\
\hline 0.001 & 0.7877 & 0.7863 \\
0.005 & 0.7874 & 0.7857 \\
0.01 & 0.7869 & 0.7849 \\
0.02 & 0.7860 & 0.7829 \\
0.03 & 0.7850 & 0.7790 \\
0.04 & 0.7841 & 0.7761 \\
0.05 & 0.7832 & 0.7737 \\
0.06 & 0.7822 & 0.7726 \\
0.07 & 0.7812 & 0.7690 \\
0.08 & 0.7802 & 0.7681 \\
0.09 & 0.7791 & 0.7648 \\
0.1 & 0.7781 & 0.7602 \\
\hline
\end{tabular}


fluctuations that are due to the random selection of nodes in the stimulation program. Irregular behavior of the results for different angles can indicate that the coefficient $\bar{\chi}$ does not depend on $\varphi$ and shows only a very small dependence on increasing $r$, which is negligible due to the small range of coefficient change for different $r$. Therefore, with an acceptable approximation, the value of the coefficient $\bar{\chi}$ can be considered 0.77; in other words, by accepting a very small error, the average coefficient $\chi$ can be considered independent of the values of $r$ and $\varphi$, therefore its value can be considered equal to 0.77 . By multiplying 0.77 in $Q$ and $Q^{\prime}$ obtained for the inner area, the values $\bar{Q}$ and $\bar{Q}^{\prime}$ can be calculated for the nodes located in the border area as follows:

$$
\begin{gathered}
\bar{Q}=\bar{\chi} \frac{M r^{2} \varphi}{2} \approx 0.77 \times \frac{M r^{2} \varphi}{2}, \\
\bar{Q}^{\prime}=\bar{\chi} \frac{M r^{2}(2 \pi-\varphi)}{2} \approx 0.77 \times \frac{M r^{2}(2 \pi-\varphi)}{2} .
\end{gathered}
$$

Therefor we have:

$$
\begin{aligned}
& \left(p_{c n}^{i}\right)_{b o}=\left(1-e^{-\bar{Q}}\right) \times \\
& \left(1-\frac{e^{-0.77 M \pi r^{2}}}{1-e^{-\bar{Q}}} \sum_{b=1}^{M-1} \sum_{N=0}^{M-1-b} \frac{\left[\left(1-\frac{\varphi}{2 \pi}\right) \bar{Q}^{\prime}\right]^{N}}{N !} \frac{\left[\left(1-\frac{\varphi}{2 \pi}\right) \bar{Q}\right]^{b}}{b !}\right) .
\end{aligned}
$$

Finally, using (4), (15) and (21), the probability of connectivity (of order 1) for an arbitrary node can be calculated as:

$$
\begin{aligned}
& p_{c n}^{i}=\left(1-e^{-Q}\right) \times(1-2 r)^{2} \times \\
& \left(1-\frac{e^{-M \pi r^{2}}}{1-e^{-Q}} \sum_{b=1}^{M-1} \sum_{N=0}^{M-1-b} \frac{\left[\left(1-\frac{\varphi}{2 \pi}\right) Q^{\prime}\right]^{N}}{N !} \frac{\left[\left(1-\frac{\varphi}{2 \pi}\right) Q\right]^{b}}{b !}\right)+ \\
& \left(1-e^{-\bar{Q}}\right) \times\left(4 r-4 r^{2}\right) \times \\
& \left(1-\frac{e^{-0.77 M \pi r^{2}}}{1-e^{-\bar{Q}}} \sum_{b=1}^{M-1} \sum_{N=0}^{M-1-b} \frac{\left[\left(1-\frac{\varphi}{2 \pi}\right) \bar{Q}^{\prime}\right]^{N}}{N !} \frac{\left[\left(1-\frac{\varphi}{2 \pi}\right) \bar{Q}\right]^{b}}{b !}\right) .
\end{aligned}
$$

\subsection{Connectivity Analysis for $k$-Connected Network}

In this section, we generalize the analyses performed in the previous subsections for the 1-connected network to the $k$ connected network. For this purpose, similar to (4), we can write:

$$
\begin{aligned}
p_{c n-k}^{i}=\left(p_{c n-k}^{i}\right)_{i n} \operatorname{Pr} & \left.n_{i} \in A_{i n}\right) \\
+ & \left(p_{c n-k}^{i}\right)_{b o} \operatorname{Pr}\left(n_{i} \in A_{b o}\right) .
\end{aligned}
$$

In (23), $\left(p_{c n-k}^{i}\right)_{i n}$ and $\left(p_{c n-k}^{i}\right)_{b o}$ can be calculated similar to (5) and (16) as:

$$
p_{c n-k}^{i}=p_{d_{k}}^{i} \times p_{a_{k} \mid d_{k}}^{i},
$$

where $p_{d_{k}}^{i}$ indicates the probability of having at least $k$ descendant nodes for node $i$, which is calculated as follows:

$$
p_{d_{k}}^{i}=\sum_{b=k}^{M-1} \frac{e^{-Q} Q^{b}}{b !}=1-e^{-Q} \sum_{b=0}^{k-1} \frac{Q^{b}}{b !} .
$$

In (24), $p_{a_{k} \mid d_{k}}^{i}$ indicates the probability of having at least $k$ antecedent nodes for node $i$ when this node has at least $k$ descendant nodes, which can be calculated as:

$$
\begin{aligned}
& P_{a_{k} \mid d_{k}}^{i}=\operatorname{Pr}\left(A_{i} \geq k \mid D_{i} \geq k\right)=1-\operatorname{Pr}\left(A_{i}<k \mid D_{i} \geq k\right) \\
& =1-\left[\operatorname{Pr}\left(A_{i}=0 \mid D_{i} \geq k\right)+\cdots+\operatorname{Pr}\left(A_{i}=k-1 \mid D_{i} \geq k\right)\right] .
\end{aligned}
$$

\subsection{Connectivity Analysis for 2-Connected Networks}

By replacing $k=2$ in (25) and (26), we will have:

$$
p_{d_{2}}^{i}=1-e^{-Q}(1+Q) \text {. }
$$

Appendix B shows that $p_{a 2 \mid d 2}^{i}$ can be written as (28), shown at the top of the next page.

Using (27) and (28) in (24), the probability of node connectivity in a 2 -connected network $(k=2)$ for nodes located in the inner area, $\left(p_{c n-2}^{i}\right)_{i n}$, and an arbitrary $\varphi$ is obtained as (29), shown at the next page.

By replacing the border area parameters in (29), the probability of node connectivity in the 2-connected network for nodes located in the border area, $\left(p_{c n-2}^{i}\right)_{b o}$, and an arbitrary $\varphi$ is as (30), shown at the next page.

By replacing (29) and (30) in (23), where $\operatorname{Pr}\left(i \in A_{\text {in }}\right)=$ $(1-2 r)^{2}$ and $\operatorname{Pr}\left(i \in A_{b o}\right)=\left(4 r-4 r^{2}\right)$, the probability of the connectivity of a typical node in the 2-connected network for an arbitrary $\varphi$ is obtained. For the sake of brevity, we have omitted writing the final formula.

When $\varphi \neq 2 \pi, p_{a_{2} \mid d_{2}}^{i}$ for the node located in the inner area can be simplified to

$$
\begin{aligned}
& p_{a 2 \mid d 2}^{i}=-\frac{e^{-M \pi r^{2}}}{1-e^{-Q}(1+Q)} \times \\
& \sum_{b=2}^{M-1} \sum_{N=0}^{M-1-b}\left\{\left(1+\frac{N \varphi}{2 \pi-\varphi}+\left(\frac{b \varphi}{2 \pi-\varphi}\right)^{2}\left(1-\frac{\varphi}{2 \pi}\right)^{b}\right) \times\right. \\
&\left.\frac{\left(\left(1-\frac{\varphi}{2 \pi}\right) Q^{\prime}\right)^{N}}{N !} \frac{\left(\left(1-\frac{\varphi}{2 \pi}\right) Q\right)^{b}}{b !}\right\}
\end{aligned}
$$

Using (27) and (31), the probability of connectivity of a node located in the inner area in the 2-connected network is obtained for $\varphi \neq 2 \pi$ as follows:

$$
\begin{aligned}
\left(p_{c n-2}^{i}\right)_{i n}= & 1-e^{-Q}(1+Q)-e^{-M \pi r^{2}} \times \\
\sum_{b=2}^{M-1} \sum_{N=0}^{M-1-b}\{ & \left(1+\frac{N \varphi}{2 \pi-\varphi}+\left(\frac{b \varphi}{2 \pi-\varphi}\right)^{2}\left(1-\frac{\varphi}{2 \pi}\right)^{b}\right) \times \\
& \left.\frac{\left(\left(1-\frac{\varphi}{2 \pi}\right) Q^{\prime}\right)^{N}}{N !} \frac{\left(\left(1-\frac{\varphi}{2 \pi}\right) Q\right)^{b}}{b !}\right\} .
\end{aligned}
$$

By replacing the border area parameters in (32), the probability of connectivity of the node located in the border strip in the 2 -connected network is calculated for $\varphi \neq 2 \pi$ as follows:

$$
\begin{aligned}
\left(p_{c n-2}^{i}\right)_{b o}= & 1-e^{-\bar{Q}}(1+\bar{Q})-e^{-0.77 M \pi r^{2}} \times \\
\sum_{b=2}^{M-1} \sum_{N=0}^{M-1-b}\left\{\left(1+\frac{N \varphi}{2 \pi-\varphi}+\left(\frac{b \varphi}{2 \pi-\varphi}\right)^{2}\left(1-\frac{\varphi}{2 \pi}\right)^{b}\right) \times\right. & \left.\frac{\left(\left(1-\frac{\varphi}{2 \pi}\right) \bar{Q}^{\prime}\right)^{N}}{N !} \frac{\left(\left(1-\frac{\varphi}{2 \pi}\right) \bar{Q}\right)^{b}}{b !}\right\} .
\end{aligned}
$$




$$
\begin{aligned}
p_{a 2 \mid d 2}^{i} & =1-\frac{1}{1-e^{-Q}(1+Q)}\left\{\sum_{b=2}^{M-1} \sum_{N=0}^{M-1-b}\left[\left(1-\frac{\varphi}{2 \pi}\right)^{N} \frac{e^{-Q^{\prime}} Q^{\prime N}}{N !}\right]\left[\left(1-\frac{\varphi}{2 \pi}\right)^{b} \frac{e^{-Q} Q^{b}}{b !}\right]\right. \\
& +\sum_{b=2}^{M-1} \sum_{N=1}^{M-1-b}\left[\frac{N \varphi}{2 \pi}\left(1-\frac{\varphi}{2 \pi}\right)^{N-1} \frac{e^{-Q^{\prime}} Q^{\prime N}}{N !}\right]\left[\left(1-\frac{\varphi}{2 \pi}\right)^{b} \frac{e^{-Q} Q^{b}}{b !}\right] \\
& \left.+\sum_{b=2}^{M-1} \sum_{N=0}^{M-1-b}\left[\frac{b \varphi}{2 \pi}\left(1-\frac{\varphi}{2 \pi}\right)^{N+b-1} \frac{e^{-Q^{\prime}} Q^{\prime N}}{N !}\right]\left[\frac{b \varphi}{2 \pi}\left(1-\frac{\varphi}{2 \pi}\right)^{b-1} \frac{e^{-Q} Q^{b}}{b !}\right]\right\} .
\end{aligned}
$$

$$
\begin{aligned}
&\left(p_{c n-2}^{i}\right)_{i n}=1-e^{-Q}(1+Q) \\
&-e^{-M \pi r^{2}}\left\{\sum_{b=2}^{M-1} \sum_{N=0}^{M-1-b}\left(\frac{\left(\left(1-\frac{\varphi}{2 \pi}\right) Q^{\prime}\right)^{N}}{N !}+\frac{N \varphi}{2 \pi}\left(1-\frac{\varphi}{2 \pi}\right)^{N-1} \frac{Q^{\prime N}}{N !}\right)\left(\frac{\left(\left(1-\frac{\varphi}{2 \pi}\right) Q\right)^{b}}{b !}\right)\right. \\
&\left.\quad+\sum_{b=2}^{M-1} \sum_{N=0}^{M-1-b}\left(\left(\frac{b \varphi}{2 \pi}\right)^{2}\left(1-\frac{\varphi}{2 \pi}\right)^{N+b-1} \frac{Q^{\prime N}}{N !}\right)\left(\left(1-\frac{\varphi}{2 \pi}\right)^{b-1} \frac{Q^{b}}{b !}\right)\right\}
\end{aligned}
$$

$$
\begin{aligned}
\left(p_{c n-2}^{i}\right)_{b o}=1 & -e^{-\bar{Q}}(1+\bar{Q}) \\
-e^{-0.77 M \pi r^{2}} & \left\{\sum_{b=2}^{M-1} \sum_{N=0}^{M-1-b}\left(\frac{\left(\left(1-\frac{\varphi}{2 \pi}\right) \bar{Q}^{\prime}\right)^{N}}{N !}+\frac{N \varphi}{2 \pi}\left(1-\frac{\varphi}{2 \pi}\right)^{N-1} \frac{\bar{Q}^{\prime N}}{N !}\right)\left(\frac{\left(\left(1-\frac{\varphi}{2 \pi}\right) \bar{Q}\right)^{b}}{b !}\right)\right. \\
& \left.+\sum_{b=2}^{M-1} \sum_{N=0}^{M-1-b}\left(\left(\frac{b \varphi}{2 \pi}\right)^{2}\left(1-\frac{\varphi}{2 \pi}\right)^{N+b-1} \frac{\bar{Q}^{\prime N}}{N !}\right)\left(\left(1-\frac{\varphi}{2 \pi}\right)^{b-1} \frac{\bar{Q}^{b}}{b !}\right)\right\}
\end{aligned}
$$

Similarly, by replacing (32) and (33) in (23), where $\operatorname{Pr}(i \in$ $\left.A_{\text {in }}\right)=(1-2 r)^{2}$ and $\operatorname{Pr}\left(i \in A_{b o}\right)=\left(4 r-4 r^{2}\right)$, the probability of connecting an arbitrary node in the 2-connected network $(k=2)$ and for $\varphi \neq 2 \pi$ is obtained. For the sake of brevity, we have omitted writing the final formula.

\section{Numerical Results and Simulation}

In this section, we present the numerical results of the analysis performed in the previous section. In other words, we draw the closed form formulas obtained in the previous section as a function of $r, \varphi$, and $M$ for the probability of connectivity of a node in the 1-connected and 2-connected networks.

Due to the lack of any experimental data to investigate the possibility of node connectivity, in this paper, computer simulation is used to evaluate the obtained analytical results. It should be noted that the results of computer simulation in this section are obtained by averaging the connectivity status of nodes in 1000 network realization (realization of $M$ random nodes with specific $\varphi$ and $r$ and with random orientation angles). In Fig. 5, the probability of connectivity for a node in the 1-connected and 2-connected networks are drawn, respectively, using equations (22) and (23) (by using Eqs. (29) and (30)) for $M=100$ and $M=500$, and for the coverage angles $2 \pi / 9, \pi / 2,3 \pi / 4$, and $2 \pi$. For the sake of comparison, in Fig. 5, the results of computer simulation are also drawn. In these diagrams, the horizontal axis is the normalized range $r$ and the vertical axis is the probability of connectivity of the node in the 1-connected $(k=1)$ and 2-connected $(k=2)$ networks.
Fig. 5 shows how increasing the number, range and coverage angle of nodes affect the probability of connectivity in 1-connected and 2-connected networks. Let us define $r_{0.99}$ as the minimum value of $r$ to achieve a connectivity probability of 0.99 . For example, based on the analytical results obtained for 500 nodes and 1-connected network with coverage angles of $2 \pi / 9, \pi / 2,3 \pi / 4$, and $2 \pi$, the value of $r_{0.99}$ is $0.2,0.13,0.1$, and 0.05 , respectively. However, in the obtained analytical results, for the coverage angle of $2 \pi / 9$ in the range $r<0.2$, the value of $r_{0.99}$ for $M=500$ nodes and the 2 -connected network does not exist and for the coverage angles $\pi / 2,3 \pi / 4$, and $2 \pi$, it is $0.15,0.12$ and 0.06 , respectively. Since increasing each of the node parameters (number, range and coverage angle) increases the cost of network realization, the obtained results help to achieve the desired probability of connectivity with the least cost. For example, in the case of 500 nodes and a coverage angle of $2 \pi$, increasing the range of the node beyond 0.06 does not bring significant improvement. In other words, at $r=0.06$, the graph has reached its saturation. In order to better compare the simulation results with the analytical results obtained in this paper and the analytical results presented in references [23] and [27], the root mean square (RMS) of error between the simulation results as a reference, and the analytical results are presented in Tables $2-5$ using the data of Fig. 5 for the 1-connected and 2-connected networks.

The results presented in Tables 2-5 clearly show that the simulation results are closer to the results of the analyses performed in this paper, compared to the analyses presented 


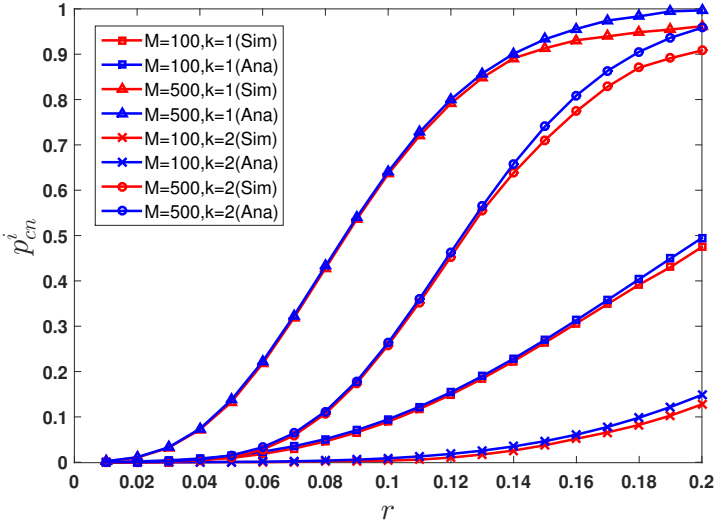

(a)

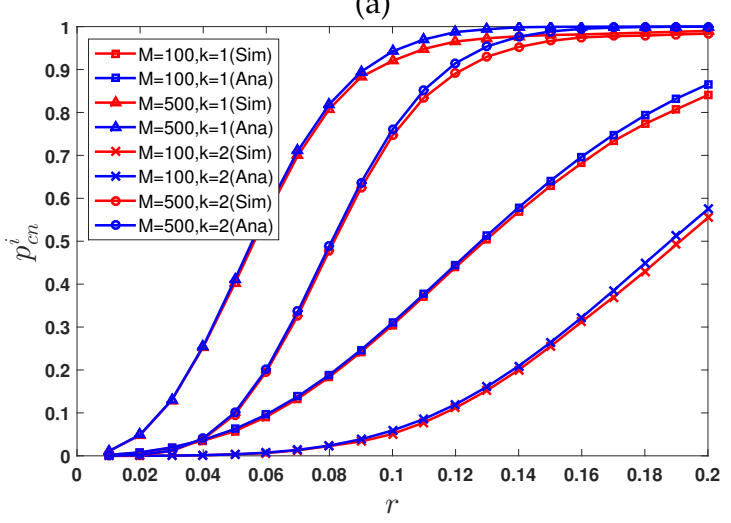

(b)

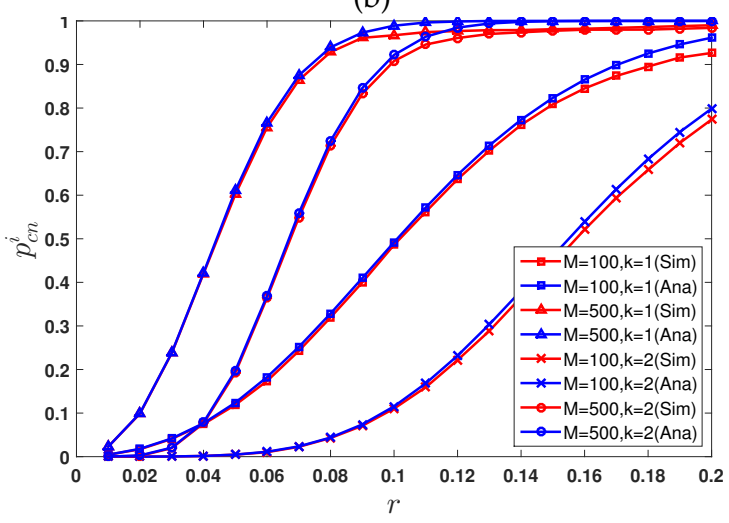

(c)

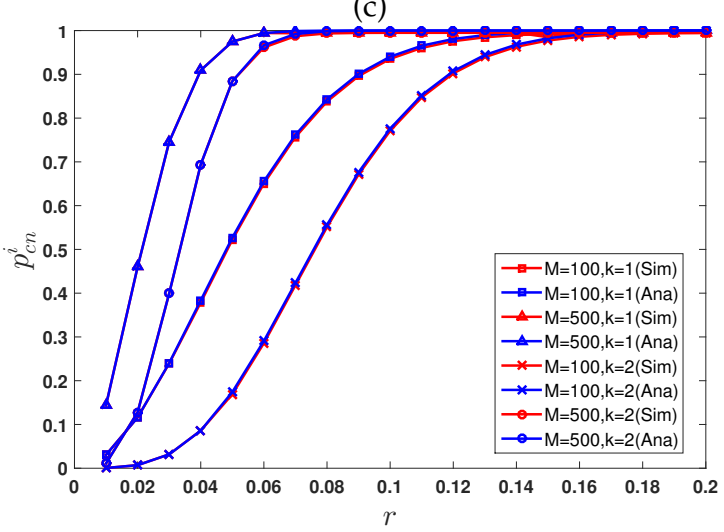

(d)

Fig. 5: Comparison of analytical results with the results obtained from computer simulation for $M=100$ and $M=500$ nodes and coverage angles (a) $2 \pi / 9$, (b) $\pi / 2$, (c) $3 \pi / 4$, and (d) $2 \pi$.
TABLE 2: Comparison of RMS of error in Fig. 5 between the simulation and the analytical results for 1-connected $(k=1)$ networks and $M=100$

\begin{tabular}{ccccc}
\hline & $\varphi=\frac{2 \pi}{9}$ & $\varphi=\frac{\pi}{2}$ & $\varphi=\frac{3 \pi}{4}$ & $\varphi=2 \pi$ \\
\hline Our work, Eq. (22) & 0.0082 & 0.0114 & 0.013 & 0.0046 \\
\hline Ref. [23] & 0.0574 & 0.0792 & 0.352 & 0.0159 \\
\hline
\end{tabular}

TABLE 3: Comparison of RMS of error in Fig. 5 between the simulation and the analytical results for 1-connected $(k=1)$ networks and $M=500$

\begin{tabular}{crrrc}
\hline & $\varphi=\frac{2 \pi}{9}$ & $\varphi=\frac{\pi}{2}$ & $\varphi=\frac{3 \pi}{4}$ & $\varphi=2 \pi$ \\
\hline Our work, Eq. (22) & 0.0184 & 0.0146 & 0.0161 & 0.0044 \\
\hline Ref. [23] & 0.0503 & 0.0437 & 0.0403 & 0.0065 \\
\hline
\end{tabular}

in [23] and [27]. For the coverage angle of $2 \pi$, the results of the mentioned references are very close to the results of our analysis. As stated in Section 3, the small value of the RMS of error for $\varphi=2 \pi$ is due to the reduction of the analyses error made in the mentioned references for this particular angle compared to other angles. It is worth noting that even at $\varphi=2 \pi$, the RMS of error between the results of the simulation and our analysis is less than the one between the results of the simulation and the mentioned references analyses.

It should be noted that although our analyses results are very close to the simulation results compared to previous works, but the numerical studies of this section showed that for relatively large values of $r$ in the range of 0.1 to 0.2 still we see a small, but increasing difference. Although this behavior may be partly due to the lack of the four square areas in the corners of the studied area, but on the other hand, it may indicate less efficiency of the models considered for connectivity analysis (such as the homogeneous Poisson point model) for the values of $r$ greater than 0.1 .

\section{CONCLUSION}

In this paper, we have analyzed the connectivity in underwater optical wireless network in which similar nodes with a certain range and coverage angle, and random surface distribution are used. In the presented analyses, we classified the nodes into two categories located in the inner area and nodes located in the border strip, and presented their connectivity analysis separately. For nodes located in the border strip, by defining the width of the border strip as the range of the node and making some assumptions, we demonstrated that the average overlap of the node sector located in the boundary strip, regardless of the range and its coverage angle is about 0.77 . By combining the mentioned analyses, we obtained closed form formulas to calculate the probability of connectivity (from orders 1 and 2) in these networks, and with the help of numerical evaluation, the effect of number, range and angle of optical node coverage on the average probability of network connectivity were studied. In addition, we compared the results of these analyses with previous analyses.

The comparison between the results of our analyses with the results of estimating the network connectivity by 
TABLE 4: Comparison of RMS of error in Fig. 5 between the simulation and the analytical results for $2-$ connected $(k=2)$ networks and $M=100$

\begin{tabular}{ccccc}
\hline & $\varphi=\frac{2 \pi}{9}$ & $\varphi=\frac{\pi}{2}$ & $\varphi=\frac{3 \pi}{4}$ & $\varphi=2 \pi$ \\
\hline $\begin{array}{c}\text { Our work, Eq. (23), } \\
\text { using Eqs. (29) and (30) }\end{array}$ & 0.0091 & 0.0098 & 0.014 & 0.0043 \\
\hline Ref. [27] & 0.0238 & 0.0948 & 0.142 & 0.027 \\
\hline
\end{tabular}

TABLE 5: Comparison of RMS of error in Fig. 5 between the simulation and the analytical results for $2-$ connected $(k=2)$ networks and $M=500$

\begin{tabular}{ccccc}
\hline & $\varphi=\frac{2 \pi}{9}$ & $\varphi=\frac{\pi}{2}$ & $\varphi=\frac{3 \pi}{4}$ & $\varphi=2 \pi$ \\
\hline $\begin{array}{c}\text { Our work, Eq. (23), } \\
\text { using Eqs. (29) and (30) }\end{array}$ & 0.0222 & 0.0157 & 0.0141 & 0.0043 \\
\hline Ref. [27] & 0.0773 & 0.066 & 0.0786 & 0.00908 \\
\hline
\end{tabular}

averaging the results of a computer simulation on 1000 independent realizations (with a random distribution of nodes in the study area) shows that the accuracy of the analyses is significantly higher in comparison with the previously presented works. For example, the numerical results of 1-connected and 2-connected analyses for 100 nodes with coverage angle of $\pi / 2$ indicate an improvement of 7th and 9th orders, respectively, compared to previous works. Finally, it should be noted that although the analysis compared to previous work is very close to the simulation results, but our numerical studies showed that still there are some differences for large normalized ranges (close to 0.2). Apart from the approximation effect used in our analyses, this behavior can be due to the inefficiency of the models considered for the analyses like the homogeneous Poisson point model for relatively large normalized ranges.

\section{APPENDIX A}

\section{Determining the COEFFICIENT $\chi$ AND $f_{\epsilon}(\epsilon)$}

Fig. 6 shows a node (the $i$-th node) with a coverage angle of $\varphi=2 \pi$ located on the border strip at the coordinate $c_{i}$. As mentioned earlier, the width of the border strip is assumed to be equal to the range of nodes and equals $r$. In the first part of this appendix, we calculate the coefficient $\chi$ (average coverage ratio of the node sector located in the border strip with the unit square area). The total area of the node sector is $A=\pi r^{2}$. The angle of the arc of the circle outside the unit square (the grey color part) can be obtained as:

$$
\zeta=2 \arccos \frac{r-\epsilon}{r}=2 \arccos \left(1-\frac{\epsilon}{r}\right) .
$$

As mentioned earlier, $\epsilon$ is the shortest distance from the inner border to the location of the node. The area of the protruding part $A^{\prime}$ of the circle (outside the unit square) can be obtained as:

$$
A^{\prime}=\pi r^{2} \frac{\zeta}{2 \pi}-\frac{1}{2} r^{2} \sin \zeta
$$

The area of the inner part of the circle $\left(A^{\prime \prime}\right)$ is obtained as:

$$
A^{\prime \prime}=A-A^{\prime}=\pi r^{2}-\left(\pi r^{2} \frac{\zeta}{2 \pi}-\frac{1}{2} r^{2} \sin \zeta\right) .
$$

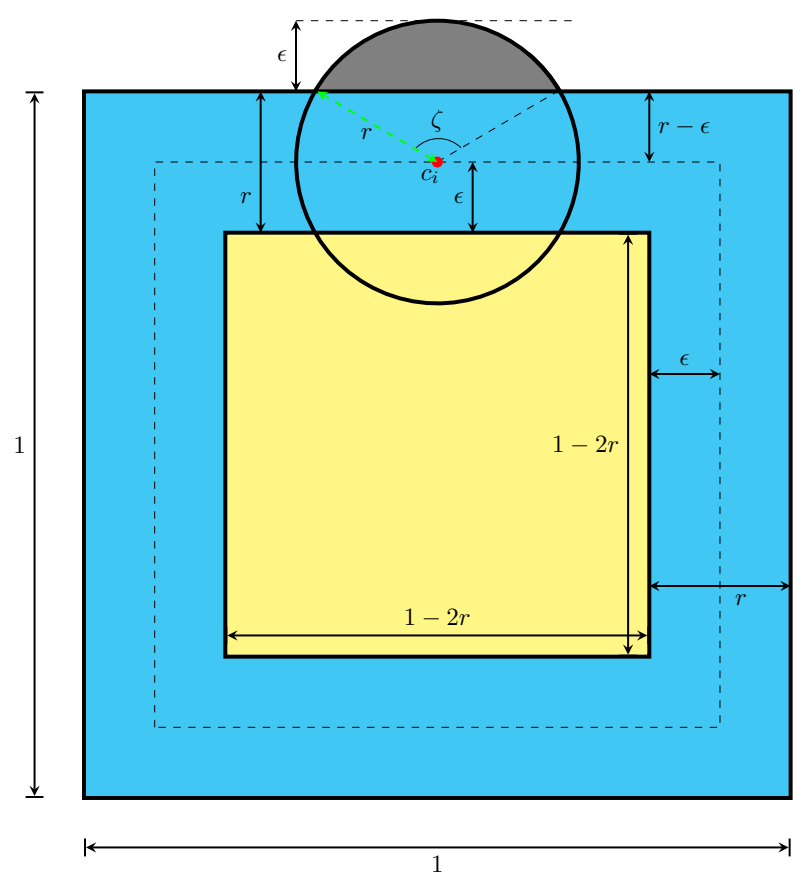

Fig. 6: Representation of a node with the coverage angles of $2 \pi$ in the border strip.

The coefficient $\chi$ is obtained by dividing $A^{\prime \prime}$ by $A$ as:

$$
\begin{aligned}
\chi=\frac{1}{\pi r^{2}}( & \pi r^{2}-\pi r^{2} \frac{2 \arccos \left(1-\frac{\epsilon}{r}\right)}{2 \pi} \\
& \left.-\frac{1}{2} r^{2} \sin \left(2 \arccos \left(1-\frac{\epsilon}{r}\right)\right)\right) .
\end{aligned}
$$

To simplify (A.1) we use relations (A.5),

$$
\left\{\begin{array}{l}
\cos \frac{\zeta}{2}=\left(1-\frac{\epsilon}{r}\right)=\sqrt{\frac{1+\cos \zeta}{2}} \\
\cos \zeta=2\left(1-\frac{\epsilon}{r}\right)^{2}-1 \\
\sin \frac{\zeta}{2}=\sqrt{\frac{1-\cos \zeta}{2}}=\frac{1}{r} \sqrt{2 \epsilon r-\epsilon^{2}} \\
\sin \zeta=2 \sin \frac{\zeta}{2} \cos \frac{\zeta}{2}=2\left(\frac{r-\epsilon}{r^{2}}\right) \sqrt{2 \epsilon r-\epsilon^{2}}
\end{array}\right.
$$

By replacing the above relations in (A.4), we obtain $\chi$ as (17).

In the following, we are looking for the answer to the question that if we assume the distribution of nodes in the border strip is uniform throughout this area, then with what probability this node will be placed on the narrow strip with a distance of $\epsilon$ from the inner border and width of $d \epsilon$. We know that the larger the $\epsilon$ is, the larger its corresponding narrow strip area will be, and therefore the more likely it is that a node in the border strip will have a larger $\epsilon$ than the smaller $\epsilon$. By defining $f_{\epsilon}(\epsilon)$ as the pdf of the random variable $\epsilon$ and note that $f_{\epsilon}(\epsilon) d \epsilon$ provides an approximation of the probability that the node will be on the narrow strip with a distance of $\epsilon$ from the inner border and width of $d \epsilon$, for the normalized problem (see Fig. 3) we have [28]:

$$
\begin{aligned}
f_{\epsilon}(\epsilon) d \epsilon & =\frac{\text { surface area of the specified narrow strip }}{\text { total surface area of border strip }} \\
& =\frac{4(1-2(r-\epsilon)) d \epsilon}{1-(1-2 r)^{2}}=\frac{1-2(r-\epsilon)}{r-r^{2}} d \epsilon .
\end{aligned}
$$


It should be noted that in the above relation, $0 \leq \epsilon \leq r$. The result obtained in (A.6) has been used in the calculations of (18).

\section{APPENDIX B Proof of (28)}

This appendix presents the details of the proof of (28). Assuming that $i$ is a node in the inner region, $p_{a_{2} \mid d_{2}}^{i}$ can be expressed as:

$$
\begin{aligned}
p_{a_{2} \mid d_{2}}^{i}=1- & \left(\operatorname{Pr}\left(A_{i}=0 \mid D_{i} \geq 2\right)+\operatorname{Pr}\left(A_{i}=1 \mid D_{i} \geq 2\right)\right) \\
=1-( & \frac{\operatorname{Pr}\left(A_{i}=0, D_{i} \geq 2, \gamma=0\right)}{\operatorname{Pr}\left(D_{i} \geq 2\right)} \\
& +\frac{\operatorname{Pr}\left(A_{i}=1, D_{i} \geq 2, \gamma=0\right)}{\operatorname{Pr}\left(D_{i} \geq 2\right)} \\
& \left.+\frac{\operatorname{Pr}\left(A_{i}=1, D_{i} \geq 2, \gamma=1\right)}{\operatorname{Pr}\left(D_{i} \geq 2\right)}\right)
\end{aligned}
$$

In the following, we will calculate the first, second and third terms in the parenthese of (B.1). Starting form the first term we have:

$$
\begin{aligned}
& \frac{\operatorname{Pr}\left(A_{i}=0, D_{i} \geq 2, \gamma=0\right)}{\operatorname{Pr}\left(D_{i} \geq 2\right)} \\
& =\frac{1}{\operatorname{Pr}\left(D_{i} \geq 2\right)} \sum_{b=2}^{M-1} \operatorname{Pr}\left(A_{i}=0, D_{i}=b, \gamma=0\right) \\
& =\frac{1}{\operatorname{Pr}\left(D_{i} \geq 2\right)} \sum_{b=2}^{M-1} \operatorname{Pr}\left(A_{i}=0 \mid D_{i}=b, \gamma=0\right) \\
& \quad \times \operatorname{Pr}\left(\gamma=0 \mid D_{i}=b\right) \operatorname{Pr}\left(D_{i}=b\right) \\
& \operatorname{Pr}\left(D_{i} \geq 2\right)=1-\operatorname{Pr}\left(D_{i}=0\right)-\operatorname{Pr}\left(D_{i}=1\right) \\
& =1-e^{-Q}(1+Q) .
\end{aligned}
$$

$$
\begin{aligned}
\operatorname{Pr}\left(A_{i}=\right. & \left.0 \mid D_{i}=b, \gamma=0\right) \\
& =\sum_{N=0}^{M-b-1} \operatorname{Pr}\left(A_{i}=0,{ }_{i} D=N \mid D_{i}=b, \gamma=0\right) \\
& =\sum_{N=0}^{M-b-1} \operatorname{Pr}\left(A_{i}=\left.0\right|_{i} D=N, D_{i}=b, \gamma=0\right) \times \\
& \operatorname{Pr}\left({ }_{i} D=N \mid D_{i}=b, \gamma=0\right) .
\end{aligned}
$$

By replacing (B.4) and (B.3) in (B.2) and using the relations in Section 3.1, the first expression in parentheses of (B.1) is obtained as follows:

$$
\begin{aligned}
\frac{\operatorname{Pr}\left(A_{i}=0, D_{i} \geq 2, \gamma=0\right)}{\operatorname{Pr}\left(D_{i} \geq 2\right)} & \frac{1}{1-e^{-Q}(1+Q)} \sum_{b=2}^{M-1} \sum_{N=0}^{M-b-1}\left[\left(1-\frac{\varphi}{2 \pi}\right)^{N} \frac{e^{-Q^{\prime}} Q^{\prime N}}{N !}\right] \times \\
& {\left[\left(1-\frac{\varphi}{2 \pi}\right)^{b} \frac{e^{-Q} Q^{b}}{b !}\right] . }
\end{aligned}
$$

To calculate the second expression in parentheses of (B.1) we do the following:

$$
\begin{aligned}
& \frac{\operatorname{Pr}\left(A_{i}=1, D_{i} \geq 2, \gamma=0\right)}{\operatorname{Pr}\left(D_{i} \geq 2\right)} \\
& =\frac{1}{\operatorname{Pr}\left(D_{i} \geq 2\right)} \sum_{b=2}^{M-1} \operatorname{Pr}\left(A_{i}=1, D_{i}=b, \gamma=0\right) \\
& =\frac{1}{\operatorname{Pr}\left(D_{i} \geq 2\right)} \sum_{b=2}^{M-1} \operatorname{Pr}\left(A_{i}=1 \mid D_{i}=b, \gamma=0\right) \\
& \quad \times \operatorname{Pr}\left(\gamma=0 \mid D_{i}=b\right) \operatorname{Pr}\left(D_{i}=b\right) . \\
& \operatorname{Pr}\left(A_{i}=1 \mid D_{i}=b, \gamma=0\right) \\
& =\sum_{N=1}^{M-b-1} \operatorname{Pr}\left(A_{i}=1,,_{i} D=N \mid D_{i}=b, \gamma=0\right) \\
& =\sum_{N=1}^{M-b-1} \operatorname{Pr}\left(A_{i}=\left.1\right|_{i} D=N, D_{i}=b, \gamma=0\right) \times
\end{aligned}
$$

With the help of the previously calculated expressions, the only new expression for calculating the second expression in parentheses of (B.1) can be calculated as follows:

$$
\begin{aligned}
& \operatorname{Pr}\left(A_{i}=\left.1\right|_{i} D=N, D_{i}=b, \gamma=0\right) \\
& =\left(\begin{array}{c}
N \\
1
\end{array}\right)\left(\frac{\varphi}{2 \pi}\right)^{1}\left(1-\frac{\varphi}{2 \pi}\right)^{N-1}=\frac{N \varphi}{2 \pi}\left(1-\frac{\varphi}{2 \pi}\right)^{N-1} .
\end{aligned}
$$

As a result, the value of the second term in parentheses of (B.1) is obtained as follows:

$$
\begin{gathered}
\frac{\operatorname{Pr}\left(A_{i}=1, D_{i} \geq 2, \gamma=0\right)}{\operatorname{Pr}\left(D_{i} \geq 2\right)}=\frac{1}{1-e^{-Q}(1+Q)} \times \\
\sum_{b=2}^{M-1} \sum_{N=1}^{M-b-1}\left[\frac{N \varphi}{2 \pi}\left(1-\frac{\varphi}{2 \pi}\right)^{N-1} \frac{e^{-Q^{\prime}} Q^{\prime N}}{N !}\right] \times \\
{\left[\left(1-\frac{\varphi}{2 \pi}\right)^{b} \frac{e^{-Q} Q^{b}}{b !}\right] .}
\end{gathered}
$$

Similarly, to calculate the third term in parentheses of (B.1), we have:

$$
\begin{aligned}
& \frac{\operatorname{Pr}\left(A_{i}=1, D_{i} \geq 2, \gamma=1\right)}{\operatorname{Pr}\left(D_{i} \geq 2\right)} \\
& =\frac{1}{\operatorname{Pr}\left(D_{i} \geq 2\right)} \sum_{b=2}^{M-1} \operatorname{Pr}\left(A_{i}=1, D_{i}=b, \gamma=1\right) \\
& =\frac{1}{\operatorname{Pr}\left(D_{i} \geq 2\right)} \sum_{b=2}^{M-1} \operatorname{Pr}\left(A_{i}=1 \mid D_{i}=b, \gamma=1\right) \\
& \quad \times \operatorname{Pr}\left(\gamma=1 \mid D_{i}=b\right) \operatorname{Pr}\left(D_{i}=b\right) . \\
& \operatorname{Pr}\left(A_{i}=1 \mid D_{i}=b, \gamma=1\right) \\
& =\sum_{N=0}^{M-b-1} \operatorname{Pr}\left(A_{i}=1,,_{i} D=N \mid D_{i}=b, \gamma=1\right) \\
& =\sum_{N=0}^{M-b-1} \operatorname{Pr}\left(A_{i}=\left.1\right|_{i} D=N, D_{i}=b, \gamma=1\right) \times
\end{aligned}
$$




$$
\begin{aligned}
\operatorname{Pr}\left(\gamma=1 \mid D_{i}=b\right) & =\left(\begin{array}{l}
b \\
1
\end{array}\right)\left(\frac{\varphi}{2 \pi}\right)^{1}\left(1-\frac{\varphi}{2 \pi}\right)^{b-1} \\
& =\frac{b \varphi}{2 \pi}\left(1-\frac{\varphi}{2 \pi}\right)^{b-1} .
\end{aligned}
$$

Same as before, as long as $N$ changes within its allowable range, the events $\left({ }_{i} D=N\right)$ and $\left(D_{i}=b, \gamma=1\right)$ can be assumed independent and we have:

$$
\begin{gathered}
\operatorname{Pr}\left({ }_{i} D=N \mid D_{i}=b, \gamma=1\right)=\frac{e^{-Q^{\prime}} Q^{\prime N}}{N !} . \\
\operatorname{Pr}\left(A_{i}=\left.1\right|_{i} D=N, D_{i}=b, \gamma=1\right) \\
=\left(\begin{array}{c}
N \\
0
\end{array}\right)\left(\frac{\varphi}{2 \pi}\right)^{0}\left(1-\frac{\varphi}{2 \pi}\right)^{N} \times\left(\begin{array}{l}
b \\
1
\end{array}\right)\left(\frac{\varphi}{2 \pi}\right)^{1}\left(1-\frac{\varphi}{2 \pi}\right)^{b-1} \\
=\frac{b \varphi}{2 \pi}\left(1-\frac{\varphi}{2 \pi}\right)^{N+b-1} .
\end{gathered}
$$

By replacing the above relations, the value of the third expression in parentheses of (B.1) is obtained as follows:

$$
\begin{gathered}
\frac{\operatorname{Pr}\left(A_{i}=1, D_{i} \geq 2, \gamma=1\right)}{\operatorname{Pr}\left(D_{i} \geq 2\right)}=\frac{1}{1-e^{-Q}(1+Q)} \times \\
\sum_{b=2}^{M-1} \sum_{N=0}^{M-b-1}\left[\frac{b \varphi}{2 \pi}\left(1-\frac{\varphi}{2 \pi}\right)^{N+b-1} \frac{e^{-Q^{\prime}} Q^{\prime N}}{N !}\right] \times \\
{\left[\frac{b \varphi}{2 \pi}\left(1-\frac{\varphi}{2 \pi}\right)^{b-1} \frac{e^{-Q} Q^{b}}{b !}\right] .}
\end{gathered}
$$

By replacing (B.5), (B.9) and (B.15) in (B.1), $p_{a 2 \mid d 2}^{i}$ is obtained as (28).

\section{ACKNOWLEDGMENTS}

This work was supported by Quchan University of Technology under a grant number 2782.

\section{RefERENCES}

[1] H. Kaushal and G. Kaddoum, "Underwater optical wireless communication," IEEE Access, vol. 4, pp. 1518-1547, Apr. 2016.

[2] E. M. Sozer, M. Stojanovic, and J. G. Proakis, "Underwater acoustic networks," IEEE J. Ocean. Eng., vol. 25, no. 1, pp. 7283, Jan. 2000.

[3] D. Pompili and I. F. Akyildiz, "Overview of networking protocols for underwater wireless communications," IEEE Commun. Mag., vol. 47, no. 1, pp. 97-102, Jan. 2009.

[4] W. W. Au, P. E. Nachtigall, and J. L. Pawloski, "Acoustic effects of the ATOC signal $(75 \mathrm{~Hz}, 195 \mathrm{~dB})$ on dolns and whales," J. of the Acous. Soc. of Am., vol. 101, May 1997.

[5] N. Saeed, A. Celik, T. Y. Al-Naffouri, and M.-S. Alouini, "Underwater optical wireless communications, networking, and localization: a survey," Ad Hoc Netw., vol. 94, 101935, Nov. 2019.

[6] C. Uribe and W. Grote, "Radio Communication Model for Underwater WSN," In 3rd Int. Conf. on New Technologies, Mobility and Security, Dec. 2009.

[7] A. I. Al-Shamma'a, A. Shaw, and S. Saman, "Propagation of electromagnetic waves at $\mathrm{MHz}$ frequencies through seawater," IEEE Trans. Antennas Propag., vol. 52, no. 11, pp. 2843-2849, Nov. 2004.

[8] S. Jaruwatanadilok, "Underwater wireless optical communication channel modeling and performance evaluation using vector radiative transfer theory," IEEE J. Sel. Areas in Commun., vol. 26, no. 9, pp. 1620-1627, Dec. 2008.

[9] F. Hanson and S. Radic, "High bandwidth underwater optical communication," Appl. Opt., vol. 47, no. 2, pp. 277-283, Jan. 2008.

[10] J. Zhang, L. Kou, Y. Yang, F. He, and Z. Duan, "MonteCarlo-based optical wireless underwater channel modeling with oceanic turbulence," Optics Communications, vol. 475, 126214, 2020.
[11] Laser Beam Propagation through Random Media, L. C. Andrews and R. L. Phillips, SPIE, 2005.

[12] M. V. Jamali, A. Chizari, and J. A. Salehi, "Performance analysis of multi-hop underwater wireless optical communication systems," IEEE Photon. Techno. Lett., vol. 29, no. 5, pp. 462-465, Mar. 2017.

[13] M. V. Jamali, F. Akhoundi, and J. A. Salehi, "Performance characterization of relay-assisted wireless optical CDMA networks in turbulent underwater channel," IEEE Trans. Commun., vol. 15, no. 6, pp. 4104-4116, Jun. 2016.

[14] M. V. Jamali and J. A. Salehi, "Performance studies of underwater wireless optical communication systems with spatial diversity: MIMO scheme," IEEE Trans. Commun., vol. 65, no. 3, pp. 11761192, Mar. 2017.

[15] P. Gjanci, C. Petrioli, S. Basagni, C. A. Phillips, L. Boloni, and Damla Turgut, "Path Finding for Maximum Value of Information in Multi-modal Underwater Wireless Sensor Networks," IEEE T. Mobile Comput., vol. 17, no. 2, pp. 404-418, May 2017.

[16] C. Wang, H.-Y. Yu, Y.-J. Zhu, “A Long Distance Underwater Visible Light Communication System With Single Photon Avalanche Diode," IEEE Photonics J., vol. 8, no. 5, Oct. 2016.

[17] R. C. Smith and K. S. Baker, "Optical properties of the clearest natural waters (200800 nm)," Appl. Opt., vol. 20, no. 2, pp. 177184, 1981.

[18] V. Chandrasekhar, W. K. Seah, Y. S. Choo, and H. V. Ee, "Localization in underwater sensor networks: Survey and challenges," In Proc. of the 1st ACM Int. Workshop on Underwater Networks, pp. 33-40, Sep. 2006.

[19] J. Luo, L. Fan, S. Wu, and X. Yan, "Research on Localization Algorithms Based on Acoustic Communication for Underwater Sensor Networks," Sensors, vol. 18, no. 1, Jan. 2018.

[20] F. Akhoundi, A. Minoofar, and J. A. Salehi, "Underwater positioning system based on cellular underwater wireless optical cdma networks," In Wireless and Opt. Commun. Conf., (WOCC), Apr. 2017

[21] N. Saeed, A. Celik, T. Y. Al-Naffouri, and M.-S. Alouini, “Underwater optical sensor networks localization with limited connectivity," In Proc. of IEEE Int. Conf. on Acoustics, Speech and Signal Processing (ICASSP), Apr. 2018.

[22] N. Saeed, A. Celik, T. Y. Al-Naffouri, and M.-S. Alouini, "Localization of energy harvesting empowered underwater optical wireless sensor networks," IEEE Trans. Wirel. Commun., vol. 18, no. 5, May 2019.

[23] Nasir Saeed , Abdulkadir Celik, Tareq Y. Al-Naffouri and Mohamed-Slim Alouini, "Performance Analysis of Connectivity and Localization in Multi-Hop Underwater Optical Wireless Sensor Networks" IEEE T. Mobile Comput., vol. 18, no. 11, pp. 26042615, Nov. 2019.

[24] R. Boluda-Ruiz, P. Rico-Pinazo, B. Castillo-Vazquez, A. GarciaZambrana, and K. Qaraqe, "Impulse response modeling of underwater optical scattering channels for wireless communication," IEEE Photonics J., vol. 12, no. 4, Aug. 2020.

[25] W. Liu, Z. Xu, and L. Yang, "SIMO detection schemes for underwater optical wireless communication under turbulence," Photon. Res., vol. 3, no. 3, pp. 48-53, Jun. 2015.

[26] X. Yi, Z. Li, and Z. Liu, "Underwater optical communication performance for laser beam propagation through weak oceanic turbulence," Appl. Opt., vol. 54, no. 6, pp. 1273-1278, Feb. 2015.

[27] U. N. Okorafor and D. Kundur, "On the Relevance of Node Isolation to the K-Connectivity of Wireless Optical Sensor Networks," IEEE T. Mobile Comput., vol. 8, no. 10, pp. 1427-1440, Oct. 2009.

[28] Probability, Statistics, and Random Processes for Electrical Engineering, A. Leon-Garcia, Peasrson Printice Hall, 2008. 


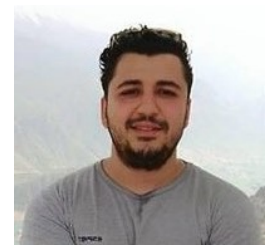

Reza Habibiyan was born in Ahwaz, Khozestan, Iran, in September 1992. He received the B.S. degree in electrical engineering from the Islamic Azad University - Shoushtar Branch, Shushtar, Iran, in 2014 and M.S. degree in electrical engineering from the Quchan University of Technology, Quchan, Iran, in 2020. His research interests include underwater optical wireless communications.

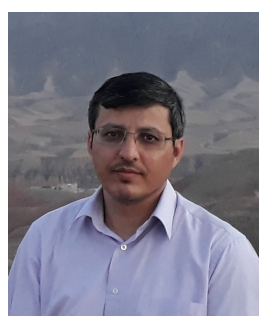

Abbasali Ghorban Sabbagh (Member, IEEE) was born in Quchan, Iran, in 1979. He received the B.S., M.S., and Ph.D. degrees in electrical engineering from the Ferdowsi University of Mashhad, Mashhad, Iran, in 2001, 2004, and 2013, respectively.

From May to September 2011, he was a member of the Optical Networks Research Laboratory (ONRL), Sharif University of Technology, Tehran, Iran. In 2016, he joined the Quchan University of Technology (QUT), Quchan, Iran, where he is currently working as an Assistant Professor. His research interests include optical CDMA, underwater optical communications, visible light communications, and free space optical communications. 